\title{
Experimental validation of the brightness distribution on the surfaces of coupled and decoupled moderators composed of $99.8 \%$ parahydrogen at the J-PARC pulsed spallation neutron source
}

Harada, Masahide; Teshigawara, Makoto; Ohi, Motoki; Klinkby, Esben Bryndt; Zanini, Luca; Batkov, Konstantin; Oikawa, Kenichi; Toh, Yosuke; Kimura, Atsushi; Ikeda, Yujiro

\section{Published in:}

Nuclear Instruments and Methods in Physics Research Section A: Accelerators, Spectrometers, Detectors and Associated Equipment

Link to article, DOI:

10.1016/j.nima.2018.06.011

Publication date:

2018

Document Version

Peer reviewed version

Link back to DTU Orbit

\section{Citation (APA):}

Harada, M., Teshigawara, M., Ohi, M., Klinkby, E. B., Zanini, L., Batkov, K., Oikawa, K., Toh, Y., Kimura, A., \& Ikeda, Y. (2018). Experimental validation of the brightness distribution on the surfaces of coupled and decoupled moderators composed of $99.8 \%$ parahydrogen at the J-PARC pulsed spallation neutron source. Nuclear Instruments and Methods in Physics Research Section A: Accelerators, Spectrometers, Detectors and Associated Equipment, 903, 38-45. https://doi.org/10.1016/j.nima.2018.06.011

\section{General rights}

Copyright and moral rights for the publications made accessible in the public portal are retained by the authors and/or other copyright owners and it is a condition of accessing publications that users recognise and abide by the legal requirements associated with these rights.

- Users may download and print one copy of any publication from the public portal for the purpose of private study or research.

- You may not further distribute the material or use it for any profit-making activity or commercial gain

- You may freely distribute the URL identifying the publication in the public portal 


\section{Accepted Manuscript}

Experimental validation of the brightness distribution on the surfaces of coupled and decoupled moderators composed of $99.8 \%$ parahydrogen at the J-PARC pulsed spallation neutron source

Masahide Harada, Makoto Teshigawara, Motoki Ohi, Esben Klinkby, Luca Zanini, Konstantin Batkov, Kenichi Oikawa, Yosuke Toh, Atsushi Kimura, Yujiro Ikeda

PII:

S0168-9002(18)30730-7

DOI: $\quad$ https://doi.org/10.1016/j.nima.2018.06.011

Reference: NIMA 60872

To appear in: $\quad$ Nuclear Inst. and Methods in Physics Research, A

Received date : 10 November 2017

Revised date: 8 April 2018

Accepted date : 6 June 2018

Please cite this article as: M. Harada, M. Teshigawara, M. Ohi, E. Klinkby, L. Zanini, K. Batkov, K. Oikawa, Y. Toh, A. Kimura, Y. Ikeda, Experimental validation of the brightness distribution on the surfaces of coupled and decoupled moderators composed of $99.8 \%$ parahydrogen at the J-PARC pulsed spallation neutron source, Nuclear Inst. and Methods in Physics Research, A (2018), https://doi.org/10.1016/j.nima.2018.06.011

This is a PDF file of an unedited manuscript that has been accepted for publication. As a service to our customers we are providing this early version of the manuscript. The manuscript will undergo copyediting, typesetting, and review of the resulting proof before it is published in its final form. Please note that during the production process errors may be discovered which could affect the content, and all legal disclaimers that apply to the journal pertain. 


\title{
Experimental validation of the brightness distribution on the surfaces of coupled and decoupled moderators composed of $99.8 \%$ parahydrogen at the J-PARC pulsed spallation neutron source
}

\author{
Masahide Harada ${ }^{\mathrm{a}, *}$, Makoto Teshigawara ${ }^{\mathrm{a}}$, Motoki Ohi ${ }^{\mathrm{a}}$, Esben Klinkby ${ }^{\mathrm{b}, \mathrm{c}}$, Luca Zanini ${ }^{\mathrm{b}}$, Konstantin \\ Batkov $^{\mathrm{b}}$, Kenichi Oikawa ${ }^{\mathrm{a}}$, Yosuke Toh ${ }^{\mathrm{a}}$, Atsushi Kimura ${ }^{\mathrm{a}}$, Yujiro Ikeda ${ }^{\mathrm{a}}$ \\ ${ }^{a}$ Japan Atomic Energy Agency, 2-4 Shirakata, Tokai-mura, Naku-gun, Ibaraki-ken, 319-1195, Japan \\ ${ }^{b}$ European Spallation Source ERIC, Box 176, S-221 00 Lund, Sweden \\ ${ }^{c}$ Technical University of Denmark, DTU Nutech, Risø Campus, Frederiksborgvej 399 , 4000 Roskilde, Denmark
}

\begin{abstract}
Liquid hydrogen moderators composed of $99.8 \%$ parahydrogen associated with light-water premoderator at the J-PARC pulsed spallation neutron source have demonstrated high performance in the extraction of high intensity cold and thermal neutron beams. In the design stage, simulations have shown not only high total neutron intensity in the coupled moderator but also a local neutron-brightness increase at its edges. The edge-effect-brightness increase is also exploited in the design of the European Spallation Source (ESS) moderators, which are also based on 99.8\% parahydrogen, but are thin (thickness: $3 \mathrm{~cm}$ ) to further increase brightness. In this study, the spatial distribution of the neutron brightness at the surface of the coupled moderator in the J-PARC pulsed spallation neutron source was directly measured to experimentally validate the calculated edge-brightness enhancement. The brightness distribution at the moderator surface predicted by Monte Carlo simulation was clearly observed. As a result, the validity of the simulation tools used in the design-optimization process of the J-PARC and ESS moderator is confirmed.
\end{abstract}

Keywords: J-PARC; Pulsed spallation neutron source; 99.8\% parahydrogen; Coupled hydrogen moderator; Premoderator; Spatial brightness; Edge-brightness enhancement

\section{Introduction}

Low-temperature hydrogen is adopted as a moderator material in MW-class spallation neutron sources for cold neutron production, such as the Japan Proton Accelerator Research Complex (J-PARC) [1] and the Spallation Neutron Source (SNS) [2-4]; hydrogen moderators have also been extensively utilized in other neutron sources, such as IPNS [5-8], ISIS [9, 10], LANSCE [11-15], CSNS [16, 17]. A recent MW spallation neutron source projects, the European Spallation Source (ESS) [18], also selected hydrogen as

*Corresponding author

Email address: harada.masahide@jaea.go.jp (Masahide Harada) 
the material of choice for the cold moderator. Although solid or liquid methane is a suitable moderator material from the viewpoint of high performance of a cold neutron source, liquid hydrogen, despite its hydrogen density, which is relatively lower than that of methane, has been the chosen solution for MWclass sources because methane could degrade easily with serious radiation damage [19-22]. Nevertheless, extensive studies on the performance of hydrogen moderators in spallation-source design have revealed that the slowing-down characteristics of parahydrogen provided high neutronic performance [23]. Moreover, the enhancement is increased when a light-water premoderator is located in the neighboring of a $99.8 \%$ parahydrogen moderator. Optimization of the geometrical size offers the higher neutronic performance, including high peak intensity with a narrow pulse and a short decay in the neutron-beam pulse [24-27]. In particular, remarkable intensity gain was achieved by introducing a moderator with a cylindrical shape with a rather large volume of parahydrogen [25]. Moreover the design studies have shown that a high-brightness region exists at positions close to the premoderator for the coupled parahydrogen moderator [24]. Although it was an very exciting finding of a significant increase neutron intensity, J-PARC has paid more attention to the high total intensity of the coupled-moderator performance as a priority.

Since the J-PARC pulsed-neutron source started operation in 2008, the importance of experimentally demonstrating the performance of the designed target-moderator-reflector assembly (TMRA) has been addressed. The essential parts of basic parameters such as intensity, pulse resolution and pulse shape have been measured to validate the high-performance design at J-PARC/MLF. Three different types of cold moderators are installed at J-PARC: a coupled-hydrogen moderator (CM), a decoupled-hydrogen moderator $(\mathrm{DM})$, and a decoupled one with a $\mathrm{Cd}$ poison $(\mathrm{PM})$, which is an absorber of slow neutrons. In these moderators, liquid hydrogen at about $20 \mathrm{~K}$ and $1.5 \mathrm{MPa}$ is circulated constantly. In Ref. [28-31] the results of first-characteristics measurement of the coupled-hydrogen moderator were reported, while in Ref. [31], measurements of one of the decoupled moderators at the J-PARC pulsed spallation neutron source were discussed.

However, the above-noted spatial-brightness enhancement of the coupled moderator currently in operation has not yet been measured due to the unavailability of a suitable detector. Meanwhile, a 2-dimensional detector, nGEM [32], coupled with a pin-hole geometry was made available and we planned an experiment to obtain precise 2-dimensional information with a high counting rate. Like the J-PARC moderators, the ESS cold moderators will use almost pure parahydrogen. The ESS team carried out a detailed study using MCNPX 2.7.0 [33], focusing on optimizing the moderator brightness, based on low-dimensional-moderator concepts $[34,35]$. This has resulted in the design of 3 -cm thick thermal and cold moderators [36, 37]. Such designs exploit the edge-brightness-increase effects predicted in Ref. [24]; because of the reduced height of the moderator, the brightness at its center is increased due to edge effects coming from both the target and the reflector sides. An experimental validation of these edge-brightness-increase effects would therefore constitute a validation of the physical concept of the ESS moderator and the simulation tools used for the design of 
the ESS moderators. Both J-PARC and ESS identified the importance of validating the brightness rise in the hydrogen cold moderator with a light-water premoderator. Experiments have been coordinated and carried out to measure the characteristics of spatial-brightness distributions at J-PARC using the viewed surface of the coupled-hydrogen cold moderator with the light-water premoderators. Additionally, we have performed measurements on the DM surrounded by decoupler, for comparison purposes. This paper describes the experimental procedure and results of measurements and simulations of spatial brightness distributions of coupled and decoupled moderators. The validity of the simulation of the brightness increase is also discussed.

\section{Experimental}

\subsection{Neutron source}

To understand the experiment, the major characteristics of the J-PARC pulsed spallation neutron source are described. At this source, a pulsed beam $(25 \mathrm{~Hz})$ of $3-\mathrm{GeV}$ protons collides with a mercury target to produce spallation neutrons. CM, PM, and DM are located below and above the target. Liquid hydrogen at about $20 \mathrm{~K}$ and $1.5 \mathrm{MPa}$ is used as the moderator material [1]. The hydrogen was assumed to be converted by a ortho-topara catalyzer [38, 39]. The experimental facility at the J-PARC pulsed spallation neutron source is shown in Fig. 1, whereas Figure 2 shows a detailed view of the TMRA. As of 2016, there were 23 beam ports and 21 instruments installed at J-PARC [40]. The moderator vessels of CM and DM are made of

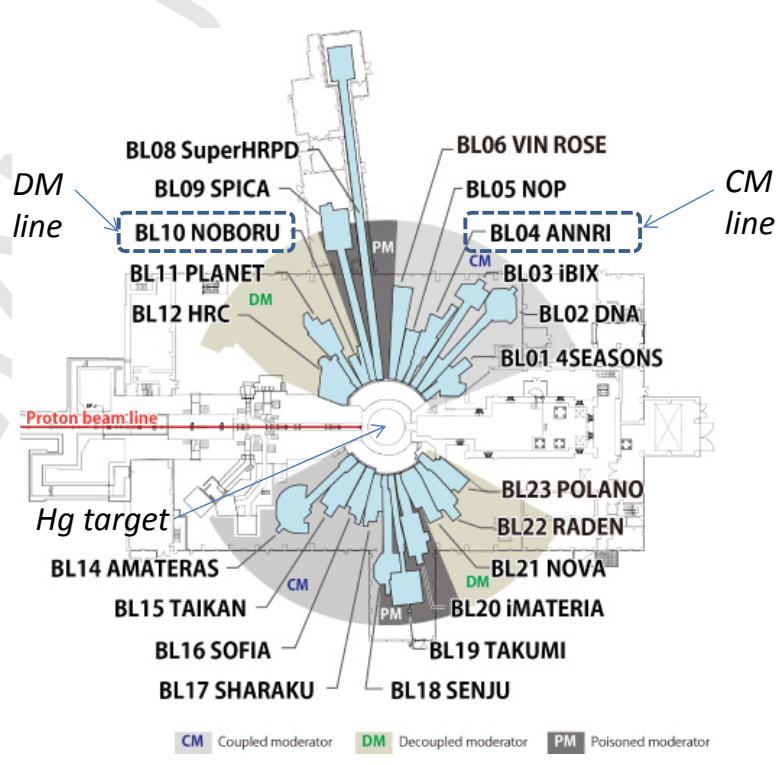

Figure 1: The experimental facility at the J-PARC outlining the beam-lines viewing CM, DM and PM as of July, 2016 [40].

aluminum-alloy. Neutrons are extracted via extraction surfaces $\left(100 \times 100 \mathrm{~mm}^{2}\right)$; premoderators surround the moderator except at the viewed surfaces. In DM and PM, a silver-indium-cadmium (AIC) decoupler [41] also surrounds the moderator to eliminate slow neutrons from the premoderator and reflector. The experiments were performed at beam line 4 (BL04; called ANNRI) [42-44] for CM and at beam line 10 (BL10; called NOBORU) [45-47] for DM. The experiments reported in this paper were performed in April 2015 using a proton beam power of $500 \mathrm{~kW}$. In the near future it is planned to ramp up the beam power to the design value of $1 \mathrm{MW}$.

\subsection{Two-dimensional detector}




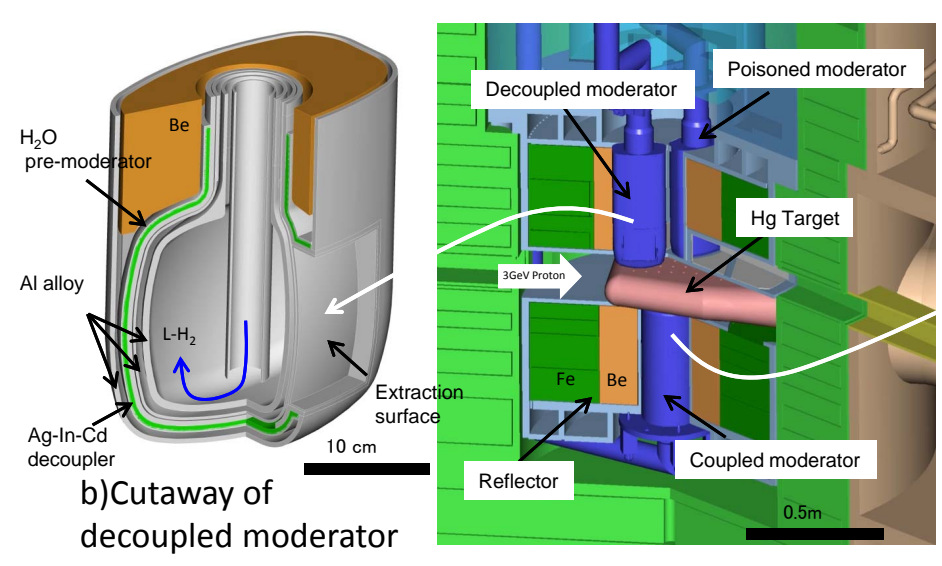

a)J-PARC target-moderator-reflector

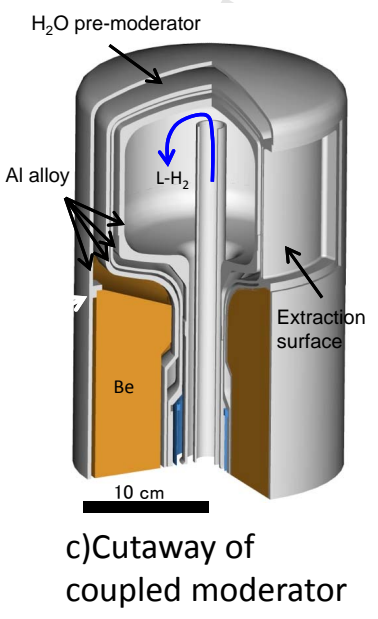

Figure 2: J-PARC target-moderator-reflector assembly. (a) Cutaway of the target-moderator-reflector assembly. b) Cutaway of the decoupled moderator. c) Cutaway of the coupled moderator.

The two-dimensional detector, nGEM, which employs a gas electron multiplier (GEM) utilizes boron foils (2.6 $\mu \mathrm{m}$ in thickness) to convert neutrons to charged particles [32]. Figure 3 shows a schematic nGEM structure. Charged particles are produced by a ${ }^{10} B(n, \alpha)$ reaction in boron coated on GEM foils. The charged particles ionize a gas $\left(70 \% \mathrm{Ar}, 30 \% \mathrm{CO}_{2}\right)$ and cause an electron avalanche in a sensitive detector with three GEM foils, thereby freeing sufficient charge to allow a signal to be collected. A voltage of $-2.65 \mathrm{kV}$ between a boron cathode and a ground (readout) in the nGEM was supplied. The detection efficiency overall follows the ${ }^{10} \mathrm{~B}$-neutronreaction cross-section, which will be relevant when com-

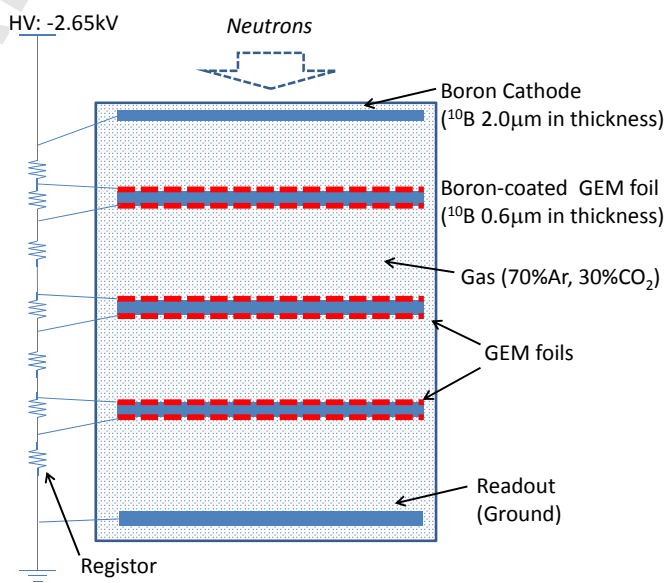

Figure 3: Outline of nGEM structure. The nGEM detector had 3 GEM foils coated with boron layers $2.6 \mu \mathrm{m}$ in thickness. The gas was a $70 \% \mathrm{Ar}+30 \% \mathrm{CO}_{2}$ mixture. paring measurements to simulations.

To correct the detector-efficiency dependence upon the ${ }^{10} B(n, \alpha)$ reaction cross section, the efficiency was estimated as a function of the neutron energy, as shown in Fig. 4 . The efficiency at $25.3 \mathrm{meV}$ is about $18 \%$. The timing resolution of the nGEM detector used is $\sim 1 \mathrm{~ns}$, whereas a spatial resolution of about $0.9 \mathrm{~mm}$ is defined by the sensitive mesh of $128 \times 128$ channels covering an active area of approximately $100 \times$ $100 \mathrm{~mm}^{2}$. The acceptable maximum count rate of the nGEM is about $10^{6} \mathrm{cps}$ (official value). An effective count rate of $1.8 \times 10^{5} \mathrm{cps}$ for the nGEM detector and was reported in Ref. [48]. The count rate in the experiments is about $10^{4} \mathrm{cps}$ at most and is much lower than the effective count rate. These specifications 
satisfy the requirements of the experiment.

In the experiment, the time-of-flight (TOF) method was adopted to distinguish neutron energies. The detector simultaneously measured the TOF of each neutron, as well as the 2-dimensional position perpendicular to the neutron beam. The flight time of the neutrons, namely, the time from the TOF-start signal (the proton-beam-extraction signal from the accelerator) to the TOF-stop signal (neutron detection) at the detector was counted by the detector.

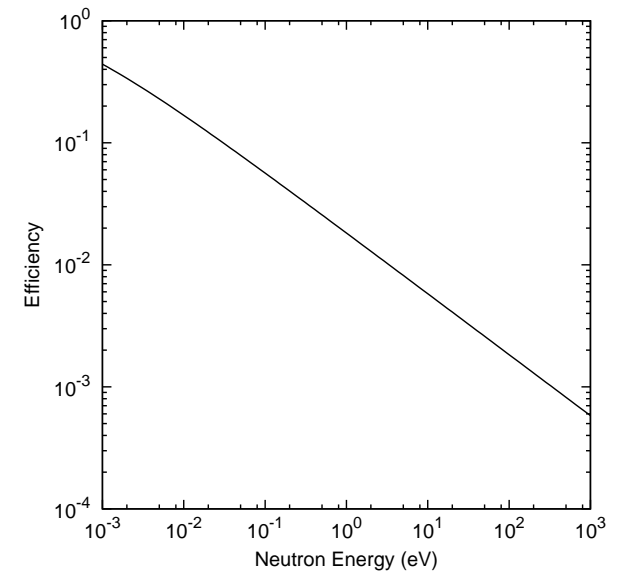

Figure 4: Predicted detector efficiency of the nGEM detector depending on the neutron reaction cross section $\left({ }^{10} B(n, \alpha)\right.$ reaction) as a function of neutron energy. The boron sheet for absorbing neutrons was $2.6 \mu \mathrm{m}$ in thickness.

To account for non-uniformity caused by the detector gain across the detection area, the detector response to a uniform neutron distribution was measured by introducing a block of polyethylene of dimensions $64 \mathrm{~mm}$ (thickness) $\times 200 \mathrm{~mm}$ (height) $\times 300 \mathrm{~mm}$ (width) in front of the detector. The distance between the polyethylene and the detector was $400 \mathrm{~mm}$. If the polyethylene block is located along each beam line, it provides uniform neutrons without time-dependence, as shown in Fig. 5 depicting a simple simulation of the neutron distribution integrated below $300 \mathrm{meV}$ after the polyethylene block by MCNPX 2.7 [33]. The result from this measurement is shown in Fig. 6,

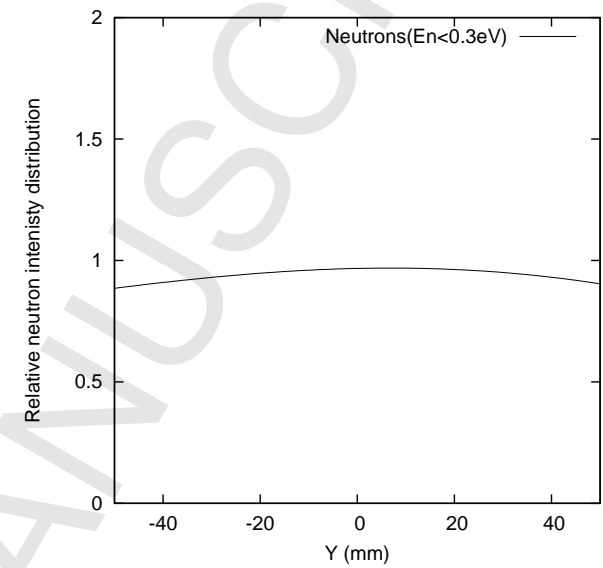

Figure 5: Simulated relative neutron intensity distribution integrated below $300 \mathrm{meV}$ as a function of the horizontal coordinate. A polyethylene block at a distance of $400 \mathrm{~mm}$ is placed in front of the detector.

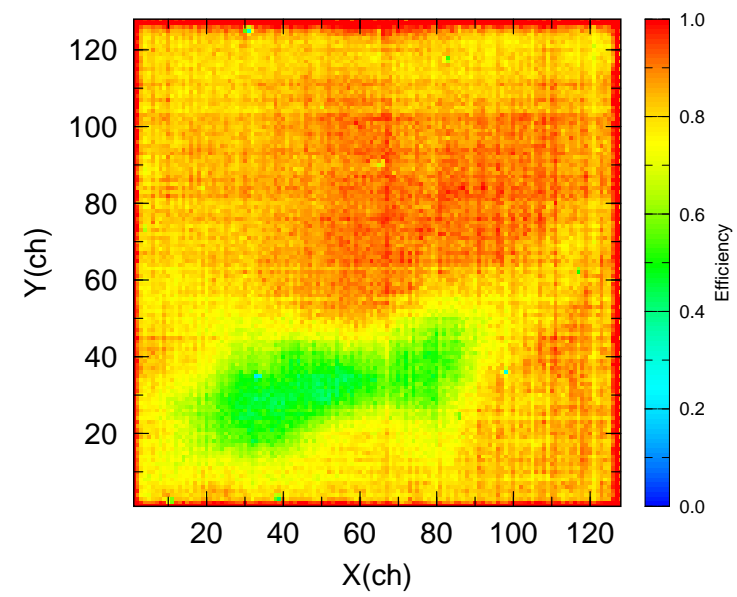

Figure 6: Detector response to a uniform neutron distribution originating from polyethylene placed in front of the detector. The distance between the polyethylene and the detector was $400 \mathrm{~mm}$.

which indicates that the neutron-detection efficiency is not uniform. The reason is that the gain (the elec- 
tron avalanche) is not uniform probably because variations in the voltage gradient among the sheets of the detector exists due to a nonuniform sheet gap. In the measurements, the measured data were corrected by this channel-dependent detector-response.

\subsection{Experimental setup}

By applying the corrections described in the previous section, a brightness map of the moderator surface is readily available from the spatial distribution observed on the detector by a pinhole collimator placed centrally between the moderator and detector. Figure 7 shows the experimental setup of the moderator, the pinhole collimator and the detector. The spatial distribution measured at the detector corresponds to the inverted image of the brightness map viewed at the surface of the moderator. The measurement data are normalized by the integrated proton-beam current measured by a monitor positioned at a proton-beamtransport line.

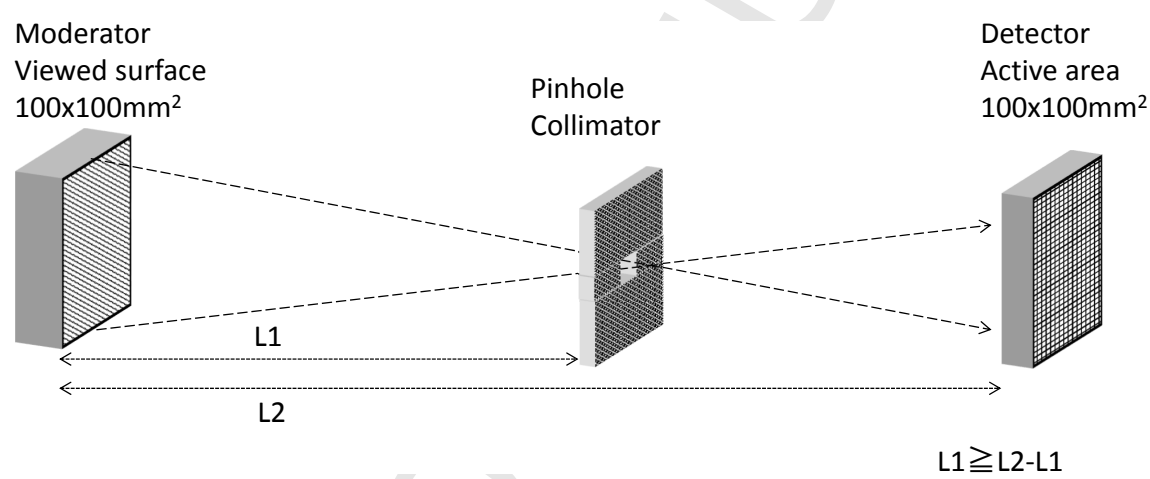

Figure 7: Schematic of the experimental setup with the pinhole geometry with the moderator and detector.

\subsubsection{Beam line BL04 from the coupled moderator}

In BL04 ANNRI, the neutron flux at $21.5 \mathrm{~m}$ downstream from the moderator is about $4.3 \times 10^{7} \mathrm{n} / \mathrm{cm}^{2} / \mathrm{s}$ in the energy range from $1.5 \mathrm{meV}$ to $25 \mathrm{meV}$ at $1 \mathrm{MW}$ power; the pulse width at an energy of $10 \mathrm{meV}$ is about $92 \mu \mathrm{s}$ [49]. The parameters of the experimental setup utilized for measurement of the coupled moderator's spatial brightness with the ANNRI beam line are shown in Table 1. The collimation of the beam is ensured by the use of a cadmium plate with a circular pinhole $3.0 \mathrm{~mm}$ in diameter, placed $20.0 \mathrm{~m}$ from the moderator. The nGEM detector is installed at $29.4 \mathrm{~m}$, resulting in a reversed image of the coupledmoderator brightness. To prevent over-illumination from the fast neutron background, a rotary collimator made of iron with a hole of $22 \mathrm{~mm}$ in diameter was introduced to the beam at a distance of $20 \mathrm{~m}$ from the moderator surface.

\subsubsection{Beam line BL10 from the decoupled moderator}

In BL10 NOBORU, the neutron flux at $14.0 \mathrm{~m}$ from the moderator is designed to be about $4.8 \times 10^{7}$ $\mathrm{n} / \mathrm{cm}^{2} / \mathrm{s}$ below $0.4 \mathrm{eV}$ at $1 \mathrm{MW}$ and the pulse widths at $10 \mathrm{meV}$ is about $33 \mu \mathrm{s}$ [49]. Although these values 


\begin{tabular}{l|l|l}
\multicolumn{2}{l}{ Table 1: Parameters of the experimental setup at BL04 and BL10 } \\
& BL04 ANNRI & BL10 NOBORU \\
\hline \hline $\begin{array}{l}\text { Distance between modera- } \\
\text { tor and pinhole }(\mathrm{m})\end{array}$ & 20.0 & 8.0 \\
\hline $\begin{array}{l}\text { Distance between modera- } \\
\text { tor and detector }(\mathrm{m})\end{array}$ & 29.4 & 13.0 \\
\hline Pinhole shape and size & $\begin{array}{l}\text { Circle shape, 3.0 } \mathrm{mm} \text { in } \\
\text { diameter }\end{array}$ & $\begin{array}{l}\text { Rectangular } \\
3.1 \mathrm{~mm} \text { in height and } \\
\text { width }\end{array}$ \\
\hline $\begin{array}{l}\text { Pinhole collimator mate- } \\
\text { rial and thickness }\end{array}$ & Cd, $1.0 \mathrm{~mm}$ & $\begin{array}{l}\text { Fe: } 350 \mathrm{~mm}, \text { Polyethy- } \\
\text { lene: } 50 \mathrm{~mm}\end{array}$
\end{tabular}

were obtained from simulations, they were validated by the measurement of a helium-3 detector with a gold-foil activation and single-crystal diffraction $[30,31]$ and are within $\pm 20 \%$ of the measured values.

The setup in place to perform the measurement of the decoupled-moderator-surface brightness using BL10 consisted of the smallest available aperture of the rotating collimator, as shown in Table 1: a pinhole of $3.1 \mathrm{~mm}$ (height) $\times 3.1 \mathrm{~mm}$ (width) positioned $8.0 \mathrm{~m}$ from the moderator surface. The rotating collimator consisted of iron $(30 \mathrm{~cm}$ in length), polyethylene $(5 \mathrm{~cm}$ in length), and iron $(5 \mathrm{~cm}$ in length) shielding. The rotating collimator reduces the background component at the detector caused by high-energy neutrons.

Neutrons passing through the pinhole was detected by the nGEM detector placed at a distance of $13.0 \mathrm{~m}$ from the moderator surface.

\section{Experimental results}

3.1. Brightness distribution on the coupled moderator

An efficiency-corrected map of the neutron brightness from the moderator can be obtained by correction based on the pinhole data, the efficiency map given by the polyethylene data in Fig. 6, and the estimated efficiency with the neutronreaction cross section in Fig. 4. This correction was applied to all measured spatial-brightnessdistribution data.

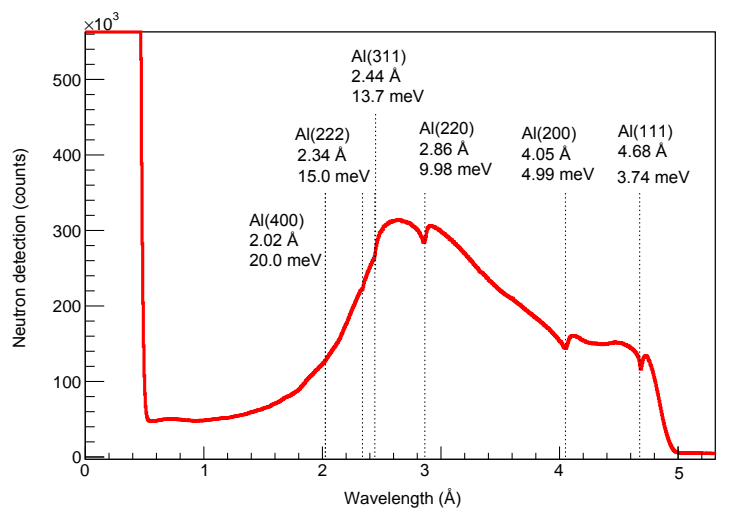

Figure 8: Wavelength spectrum derived from time-of-flight measurements at BL04. Reference values of the aluminum main Bragg edges are indicated by dashed lines. The total effective aluminum thickness is $7 \mathrm{~mm}$ between the moderator and the detector.0. High count components seen below $0.5 \AA$ are caused by higher energy neutrons than cut-off energy of $\mathrm{Cd}$.

The aim of this experiment is to provide brightness maps (i.e., spatial distributions of neutron brightness across the surfaces of the moderators) for various neutron energy regions; thus, the individual neutron energy must be accurately determined. The neutron energy was measured indirectly through its detector timestamp, which is interpreted as the neutron TOF and, given the well-known flight length, converted to an 


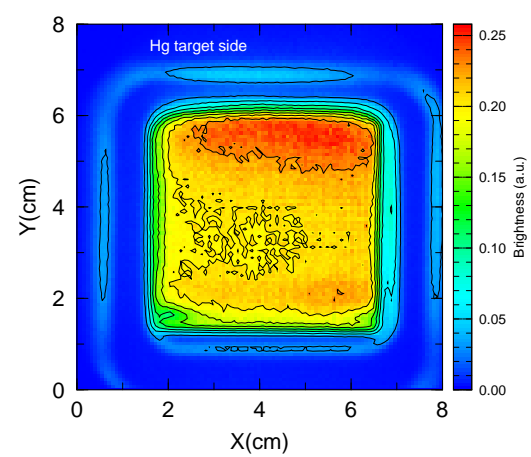

(a) Energy region: $0.8-5 \mathrm{meV}$

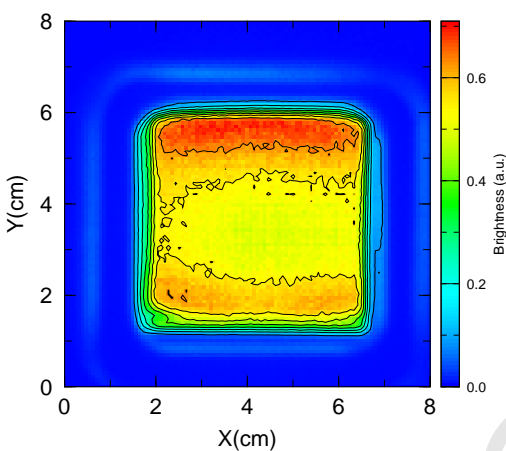

(b) Energy region: 5-10 meV

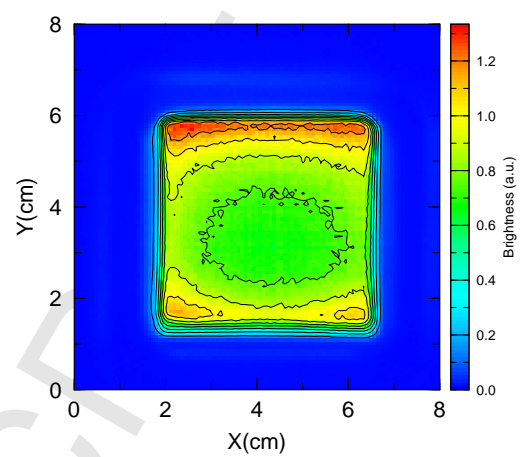

(c) Energy region: 10-100 meV

Figure 9: Measured spatial distributions of the brightness of the coupled J-PARC moderator, as seen from the ANNRI instrument (BL04). Energy regions are (a)0.8 - $5 \mathrm{meV},(\mathrm{b}) 5-10 \mathrm{meV}$ and (c) $10-100 \mathrm{meV}$. The Hg-target side is in the upper side.

energy measurement. Figure 8 shows the wavelength spectrum measured by the nGEM in BL04. Reference values of the aluminum main Bragg edges are also indicated in this figure to confirm the neutron energy by comparison with the Bragg edges. Because the reference values of the Bragg edges match the edges seen in the data well, we conclude that the TOF-start-timing signal at the accelerator and the TOF-stop-timing signal at the detector are accurate.

The spatial brightness distributions shown in Fig. 9 are obtained at the detector positions for three selected energy regions, namely $0.8-5 \mathrm{meV}$, 5-10 meV and 10-100 meV, which correspond to the cold region, the cold-thermal region, and the thermal region, respectively. The distributions for those regions in Fig. 9 clearly show higher intensity on the Hg-target side and the neutronproduction-target side. A less pronounced highintensity region is visible on the reflector side, opposite to the $\mathrm{Hg}$ target.

\subsection{Brightness distribution on the decoupled mod-} erator

Following the same procedure as for the CM

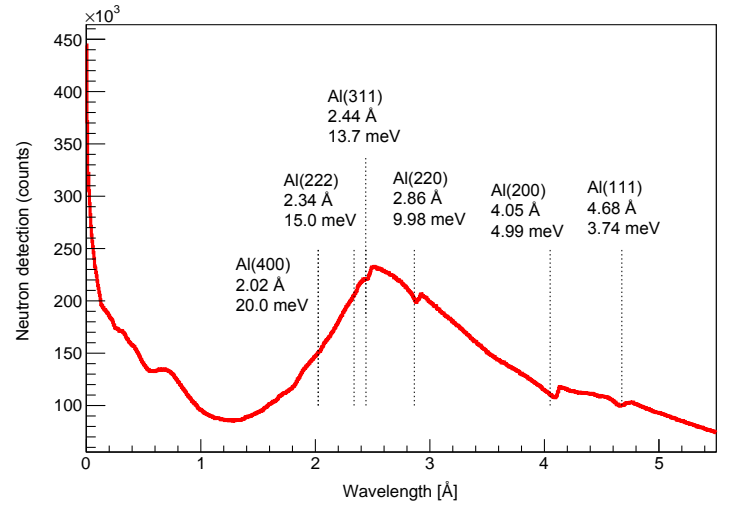

Figure 10: Wavelength spectrum derived from time-of-flight measurements at BL10. The reference values of the aluminum main Bragg edges are indicated by dashed lines. The total effective aluminum thickness between the moderator and the detector is $7 \mathrm{~mm}$.

measurements (BL04), the DM measurements (BL10) were also corrected for detector efficiency. As described in Sec. 3.1, Figure 10 shows a wavelength spectrum measured at BL10 by the detector, which is viewing the DM. The reference values of the aluminum main Bragg edges are also indicated in this figure to confirm the neutron energy by comparison with the Bragg edges. As with the measured TOF at BL04, Fig. 10 confirms 


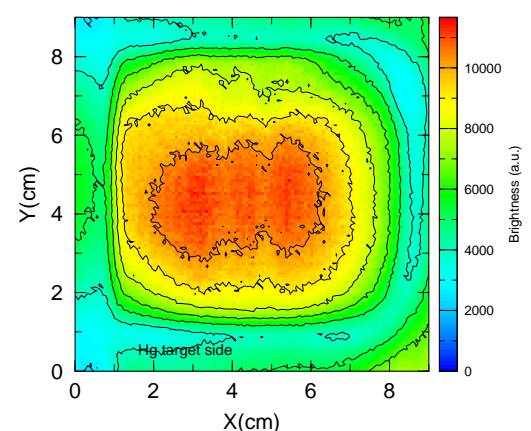

(a) Energy region: $0.8-5 \mathrm{meV}$

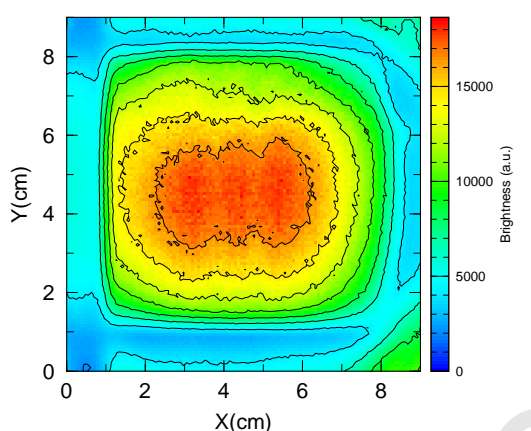

(b) Energy region: 5-10 meV

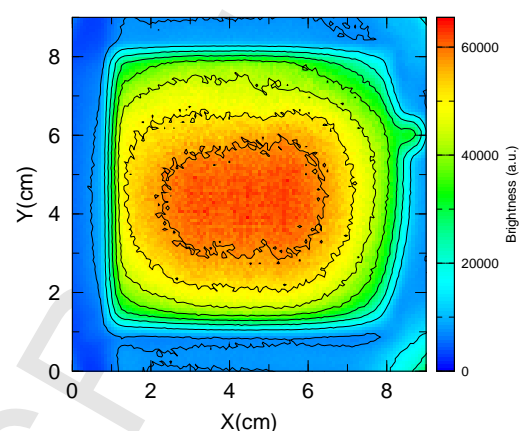

(c) Energy region: 10-100 meV

Figure 11: Measured spatial distribution of the brightness of the decoupled J-PARC moderator as seen from the NOBORU instrument (BL10). Energy regions are (a) $0.8-5 \mathrm{meV}$, (b) $5-10 \mathrm{meV}$ and (c) $10-100 \mathrm{meV}$. The $\mathrm{Hg}$ target side is on the lower side.

that the detector is also timed accurately in the case of the DM measurements.

The spatial-brightness distributions shown in Fig. 11 are obtained at the detector positions for three energy regions, $0.8-5 \mathrm{meV}, 5-10 \mathrm{meV}$ and $10-100 \mathrm{meV}$, corresponding to the cold region, the cold-thermal region, and the thermal region, respectively. In contrast to the CM case, the distributions for all energy regions are clearly more intense at the center. The two vertical stripes of lower intensity are due to shadows of the hydrogen inlet pipe. The slightly higher than average intensity at around $\mathrm{X}=8 \mathrm{~cm}$ and $\mathrm{Y}=6 \mathrm{~cm}$ corresponds to a decoupler deficit for welding of the aluminum alloy.

\subsection{Experimental error}

The statistical error in the brightness at each pixel and each energy region is below $\pm 1 \%$. We considered two systematic error sources, namely the position of the detector and the collimator relative to the moderator, and the stability of proton-beam monitor, which contribute errors of $\pm 1 \%$ and $\pm 5 \%$, respectively. The correction factor for efficiency is not counted for the systematic error. The total error of the brightness is assumed to be $\pm 7 \%$ at most.

The spatial resolution is mainly limited by the pinhole size. As the pinhole sizes in the experiment are $3.0 \mathrm{~mm}$ in diameter at BL04 and $3.1 \times 3.1 \mathrm{~mm}^{2}$ at BL10, the spatial resolution is considered to be about $5 \mathrm{~mm}$.

\section{Discussion}

\subsection{Validation of the simulations}

The simulations of the brightness distributions of CM and DM are performed using MCNPX 2.7.0, which is used in the design of the J-PARC spallation-neutron source and the ESS TMRA. The TMRA model [31] in the simulation is based on the engineering design of the neutron source and accounts for all of the source's main features and, in particular, the beam-line components of BL04 and BL10: a mercury 


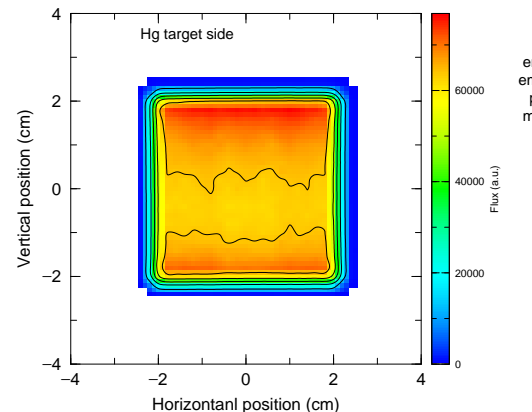

(a) Energy region: $0.8-5 \mathrm{meV}$

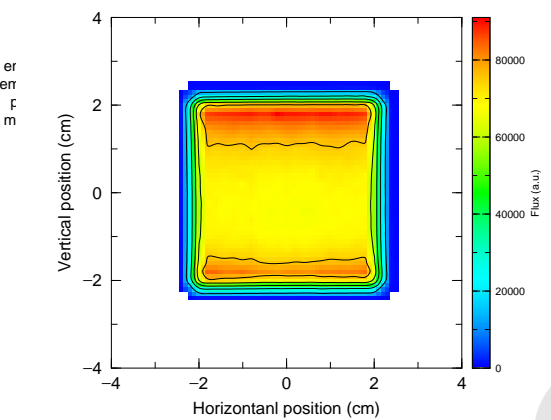

(b) Energy region: 5-10 meV

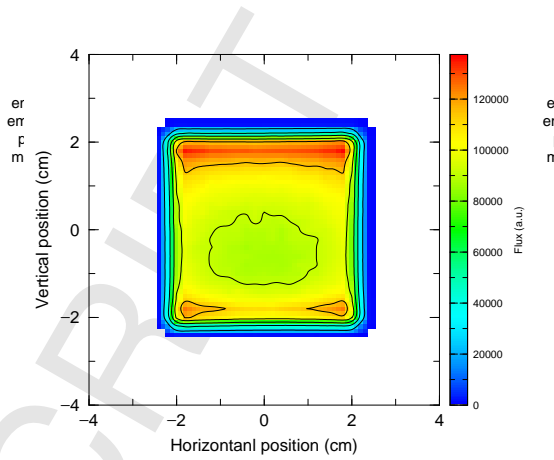

(c) Energy region: 10-100 meV

Figure 12: Simulated spatial distribution of brightness for CM (BL04). Energy ranges are (a)0.8 - 5 meV, (b) $5-10$ meV and (c) $10-100 \mathrm{meV}$. The target side is at the top part of the Y-coordinate axis.

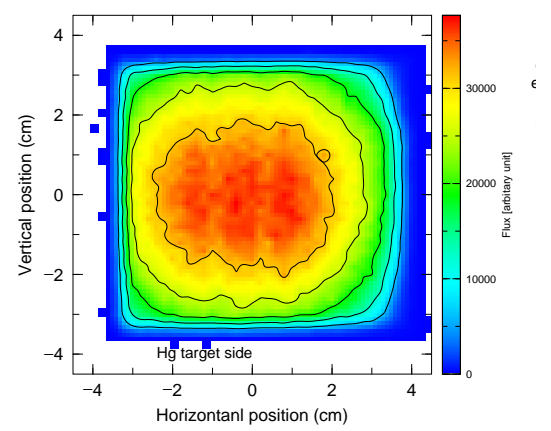

(a) Energy region: $0.8-5 \mathrm{meV}$

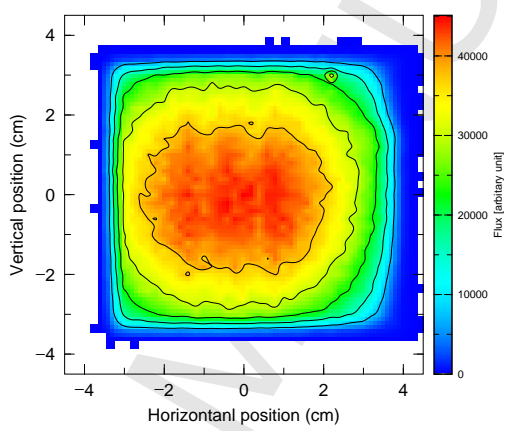

(b) Energy region: 5-10 meV

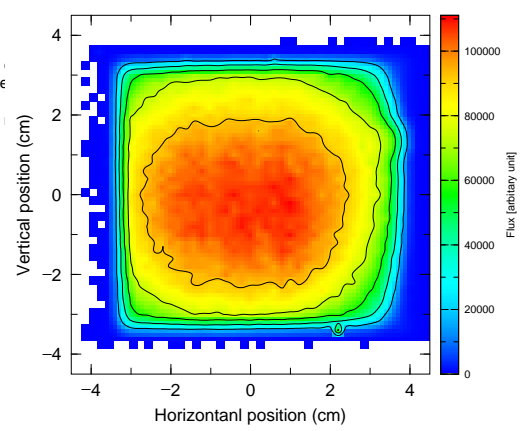

(c) Energy region: 10-100 meV

Figure 13: Same as Fig. 12 but for DM (BL10). Energy ranges are (a)0.8 - $5 \mathrm{meV}$, (b) $5-10 \mathrm{meV}$ and (c) $10-100 \mathrm{meV}$. The target side is at the bottom part of the Y-coordinate axis.

target, moderators, reflectors, shielding, and ducts for neutron-beam extraction and pinhole collimators. The positions of the pinhole and the detector were the same as shown in Table 1, corresponding to the experimental setup. The JENDL-3-evaluated cross-section library $[50,51]$ and the evaluated scattering kernel of parahydrogen and orthohydrogen listed in Ref. [52] were used. The reason to choose the kernel [52] was that the kernel was validated and was good agreement with the experimental data.[31] To calculate the brightness at the moderator surface, a radiography tally equipped in MCNPX was used.

Figures 12 and 13 show simulation results of the brightness distributions obtained at the detector positions in BL04 and BL10, respectively. Comparing with Figs. 9 and 11, it can be seen that the simulations are in good agreement with the measurements. The simulations also show the high intensities on the Hg-target side for CM and toward the center part for DM in all 3 energy regions. When the brightness distributions between the simulations and the measurements are observed in detail, the brightness outside the moderatorviewed surface in the measurement is higher. This effect is thought to be due to duct functioning as a neutron guide. In this case, the aluminum-alloy reflector vessel near the moderator, a shutter duct $(2.3 \sim$ 
$4.3 \mathrm{~m}$ from the moderator) and a duct at biological shields ( $4.3 \sim 7.0 \mathrm{~m}$ from the moderator) reflect neutrons downstream, and such effects were not modeled in the simulations.

The slightly higher-intensity region shown at the upper-right of Figs. 11(c) and 13(c) (at X $=8.5 \mathrm{~cm}$ and $\mathrm{Y}=6.5 \mathrm{~cm}$ in Fig. 11) is due to a gap of $1 \mathrm{~cm}$ in the decoupler for the welding of the moderator vessel; the two lower-intensity vertical lines at the center of Figs. 11(a) (c) and 13(a) (c) (at X $=4.0 \mathrm{~cm}$ and $\mathrm{X}=5.5 \mathrm{~cm}$ in Fig. 11) indicate the presence of inlet pipes.

Figure 14 shows the vertical cross-sectional neutron brightnesses of CM and DM for the measurements and the simulations. It also clearly indicates that the brightness distributions of both simulations and measurements show high intensity on the Hg-target side for $\mathrm{CM}$ in all 3 energy regions. Concerning the DM, the highest brightness is found at the center of the DM in all 3 energy regions. The intensity gains of the Hg-target side compared to the center part of the CM (edge-brightness enhancement) are about 1.2 at $0.8-5 \mathrm{meV}, 1.4$ at $5-10 \mathrm{meV}$ and 1.7 at $10-100 \mathrm{meV}$ in both the simulations and measurements. The duct reflection was not considered in the simulation but can be seen at $\pm 3.0 \mathrm{~cm}$ from the center in the measurements.

Overall, the simulation tools used in the design of the J-PARC spallation neutron source and of the ESS moderators are found to be reliable.

\subsection{Premoderator effect}

From the comparison between Figs. 9 and 11, the premoderator effect upon the cold-neutron density in the moderator is clear. In the CM, the distributions for all 3 energy regions in Fig. 9 clearly show higher intensities on the Hg-target side. This is explained as follows: the mean free path of a thermal neutron (10$100 \mathrm{meV}$ ) in parahydrogen is about $1.2 \mathrm{~cm}$, and the energy transfer for a spin-flip interaction of a thermal neutron with a parahydrogen molecule $(=14.7 \mathrm{meV}$, mainly) is significant compared to the scattering. Thus, only a few interactions (likely to happen close to the surface at which the neutron entered) are required for a thermal neutron to become cold enough to make the parahydrogen transparent, creating a high-intensity region close to the target. Moreover, a high-intensity region is also visible at the opposite side of the $\mathrm{Hg}$ target. This phenomenon can be understood by the same arguments, only in this case, the source is the Be reflector (i.e., the less intense source).

In contrast to the $\mathrm{CM}$ case, the highest intensities are clearly found at the centers of the DM distributions for all 3 energy regions as shown in Fig. 11. This can be explained as follows: Neutrons with energies above $1 \mathrm{eV}$ that enter the moderator are largely unaffected by the absorbing material in the decoupler. However, although the premoderated neutrons in the CM case needed only a few scatterings to reach the cold region, in the DM case several scatterings are required to down-scatter to the cold region, resulting in a deeper penetration into the moderator for several scatters. The edge effect observed in the CM is not found here due to the presence of the decoupler. The edge brightness enhancement is higher at 10-100 meV than those 

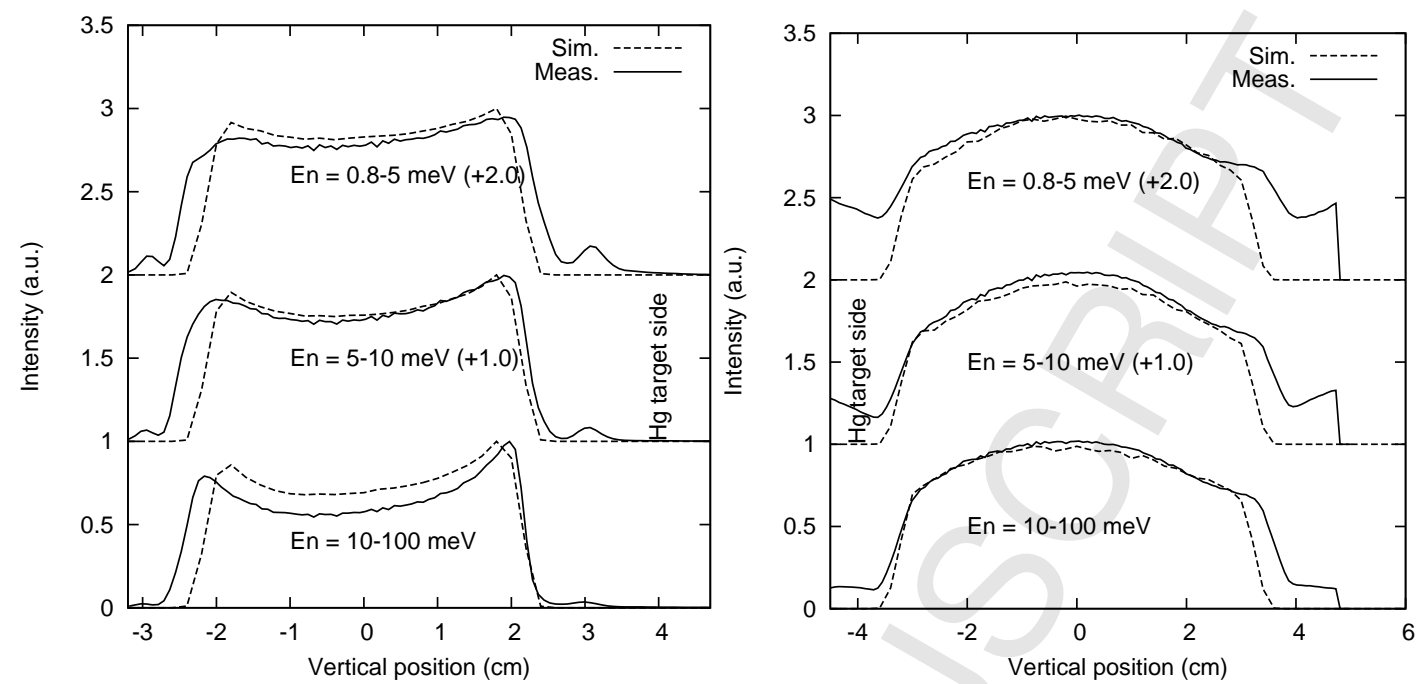

Figure 14: Vertical distribution of neutron brightness for CM (left) and DM (right) at the center position. Solid lines are measured data and dashed lines are simulated ones. Three different energy regions (0.8 - 5, 5 - 10 and 10 - 100 meV) are shown. These data are normalized to be 1.0 at the peak in each data.

at $0.8-5 \mathrm{meV}$ or $5-10 \mathrm{meV}$. It is understood that this energy dependence is related to the parahydrogen cross section which is low at lower energy.

\section{Conclusions}

Based on the pinhole imaging technique the spatial distribution of brightness across the surfaces of the coupled and the decoupled parahydrogen moderators have been measured at the BL04 and BL10 beam-lines. The results for the coupled moderator show high-intensity regions on the Hg-target side and on the reflector side. The peak brightness at the Hg-target side is increased by a factor of 1.2-1.7 compared with the center of the moderator. This behavior is both qualitatively and quantitatively reproduced by simulations and is explained by the special characteristic of the parahydrogen cross-section. No such effect is observed in the case of the decoupled moderator. Comparison with experimental measurements was used to validate the simulation, thereby validating the designs of the J-PARC spallation neutron source and the ESS spallation neutron source.

\section{Acknowledgments}

The neutron experiment at the Materials and Life Science Experimental Facility of the J-PARC was performed under a user program (Proposal No. 2015P0801). We would like to appreciate the experiment supports at ANNRI and NOBORU.

\section{References}

[1] Y. Ikeda, et al., Nucl. Instr. Meth. A 600 (2009) 1. 
[2] E.B. Iverson, et al., J. Neut. Res. 11 (2003) 83.

[3] E.B. Iverson, et al., Trans. American Nucl. Soc. 89 (2003) 673.

[4] W. Lu, et al., J. Nucl. Mat. 377 (2008) 268.

[5] J.M. Carpenter, el al., Nucl. Instr. Meth. A 234 (1985) 542.

[6] E.B. Iverson, et al., Proc. Inter. Work. Cold Neut. Sources (1997) 283.

[7] B.J. Micklich, et al., Proc. Collab. Adv. Neut. Source XVI (ICANS-XVI) (2003) 719.

[8] B.J. Micklich, et al., Proc. Collab. Adv. Neut. Source XVII (ICANS-XVII) (2006) 463.

[9] G.M. Allen, et al., Proc. Inter. Work. Cold Neut. Sources (1997) 43.

[10] S.M. Bennington, et al., J. Neut Res. 11 (2003) 93.

[11] E.J. Pitcher, et al. Proc. 3rd Inter. Topical Meeting Nucl. App. Acc. Technol. (1999) 527.

[12] G.J. Russell et al., Proc. Collab. Adv. Neut. Source XVI (ICANS-XVI) (2003) 753.

[13] G. Muhrer et al., Nucl. Instr. Meth. A 527 (2004) 531.

[14] G. Muhrer et al., Nucl. Instr. Meth. A 536 (2005) 154

[15] M. Mocko et al., Nucl. Instr. Meth. A 704 (2013) 27.

[16] Q.W. Yan, et al. J. Nucl. Mat 343 (2005) 45.

[17] F.W. Wang, et al. Sci. China-phys. Mech. Astr. 56, (2013) 2410.

[18] European Spallation Neutron Source (ESS) home page, https://europeanspallationsource.se/.

[19] J.M. Carpenter, Nature 330 (1987) 358.

[20] E. Shabalin, et al., Rad. Phys. Chem. 67 (2003) 315.

[21] E. Kulagin, et al., Nucl. Instr. Meth. B 215 (2004) 181.

[22] E. Shabalin, et al., Nucl. Instr. Meth. B 266 (2008) 5126.

[23] N. Watanabe, et al., J. Neut. Res., 11 (2003) 13.

[24] T. Kai, et al., Nucl. Instr. Meth. A 523 (2004) 398.

[25] T. Kai, et al., Nucl. Instr. Meth. A 550 (2005) 329.

[26] M. Harada, et al., Nucl. Instr. Meth. A 539 (2005) 345.

[27] M. Harada, et al., Nucl. Instr. Meth. A 574 (2007) 407.

[28] F. Maekawa, et al.,Nucl. Instr. Meth. A 620 (2010) 159.

[29] K. Oikawa, et al., Nucl. Instr. Meth. A 589 (2008) 310.

[30] K. Oikawa, et al., JPS Conf. Proc. 1, (2014) 014012.

[31] M. Harada, et al., Prog. Nucl. Sci. Technol. 2 (2011) 872.

[32] S. Uno et al., Physics Procedia 26 (2012) 142.

[33] L.S. Waters, et al., AIP Conf. Proc. 896 (2007) 81.

[34] K. Batkov, et al., Nucl. Instr. Meth. A 729 (2013) 500.

[35] F. Mezei et al., J. Neut. Res. 17 (2014) 101.

[36] L. Zanini, et al., The neutron moderators at the European Spallation Source, Proc. AccApp 15 Conf. (2015).

[37] L. Zanini, et al., The neutron moderators for the European Spallation Source, Proc. Inter. Collab. Adv. Neut. Source XXII (ICANS-XXII) (2017).

[38] H. Tatsumoto, et al., AIP Conf. Proc. 1573 (2014) 66.

[39] M. Teshigawara, et al., Nucl. Instr. Meth., B 368 (2016) 66.

[40] MLF home page at J-PARC, http://j-parc.jp/researcher/MatLife/en/instrumentation/ns.html

[41] M. Teshigawa et al., J. Nucl. Mater., 343, (2005) 154.

[42] M. Oshima, et al., Proc. Inter. Conf. ND 2007 (2007) 603.

[43] Y. Kiyanagi, et al., J. Korean Phys. Soc, 59 (2011) 1781.

[44] M. Igashira, et al., Nucl. Instr. Meth., A 600 (2009) 332.

[45] K. Oikawa, et al., Nucl. Instr. Meth., A 589 (2008) 310.

[46] F. Maekawa, et al., Nucl. Instr. Meth., A 600 (2009) 335.

[47] M. Harada, et al., JPS Conf. Proc., 1 (2014) 014015.

[48] J.D. Parker, et al.,"Development of Energy-Resolved Neutron Imaging Detectors at RADEN", Submitted to Proc. NOP 2017, (2017).

[49] Nominal characteristics of pulsed spallation neutron source in J-PARC, http://www.j-parc.jp/researcher/MatLife/ja/instrumentation/index.html

[50] T. Nakagawa, K. Shibata, S. Chiba, J. Nucl. Sci. Technol., 32 (1995) 1259.

[51] K. Shibata, et al., J. Nucl. Sci. Technol., 34 (1997) 1171.

[52] R.E. MacFarlane, Cold-moderator scattering kernel methods, LA-UR-98-655, Los Alamos National Laboratory, USA (1998). 